\title{
El desarrollo de los Talleres de Evaluación, Programación y Capacitación educativa TEPCE y su aplicabilidad en el aula multinivel del preescolar
}

\author{
Sandra Ivania Hernández Pérez ${ }^{1}$ \\ Hosny Edith Cuadra Pineda ${ }^{2}$ \\ Kenia María González Vallecillo ${ }^{3}$ \\ Juana de Jesús Benavides Laguna ${ }^{4}$
}

\section{RESUMEN}

Este trabajo trata sobre el Desarrollo de los Talleres de Evaluación, Programación y Capacitación Educativa (TEPCE), y su aplicabilidad en el aula de clases multinivel del preescolar Janeth Rodríguez del municipio de Pueblo Nuevo, Estelí, en el segundo semestre del año 2014. En esta investigación se analiza la relación que existe entre el desarrollo de los TEPCE y la aplicación en el aula de clases, como facilita los procesos de planificación y evaluación y su incidencia en el proceso de enseñanza aprendizaje de los niños y niñas de Educación Inicial. El método utilizado fue el cualitativo con enfoque descriptivo el cual permitió diseñar instrumentos de recolección de la información apropiados para analizar el fenómeno de acuerdo a la realidad estudiada, entre estos esta la entrevista, observación y grupo focal. Al concluir el presente estudio se determinó que no existe relación entre las estrategias metodológicas orientadas en el desarrollo de los TEPCE y las puestas en práctica en el aula de clase, debido a que en el TEPCE no se están orientando nuevas estrategias, además se llegó a la conclusión que la principal desventaja en el desarrollo de los TEPCE es el tiempo, ya que en el tiempo asignado para su desarrollo se dan otras actividades.

Palabras clave: Enseñanza, aprendizaje, TEPCE, estrategias metodológicas, aplicabilidad.

Recibido: 14 de enero de 2015

Aceptado: 24 de junio de 2015

1 UNAN-Managua/FAREM-Estelí: Correo Electronico: zabddyn@yahoo.com 2 UNAN-Managua/FAREM-Estelí: Correo Electronico: cuadrapedagogy@gmail.com 3 UNAN-Managua/FAREM-Estelí: Correo Electronico: gkeniamaria@yahoo.com 4 Maestra de Educación Primaria, Licenciada en Ciencias de la Educación con mención en Preescolar, Máster en Formador de Formadores de Educación Primaria y Básica. Docente del departamento de Ciencias de la Educación y humanidades, UNAN-Managua/FAREM-Estelí. Correo Electrónico: juabln@yahoo.es 


\title{
The development of the evaluation workshops, training and educational programming TEPCE and its applicability in multilevel preschool classrooms
}

\begin{abstract}
This paper deals with the development of the Evaluation Workshops, Programming and Educational Training (TEPCE), and its applicability in the multilevel preschool classroom at Janeth Rodriguez preschool of the municipality of Pueblo Nuevo, Estelí, in the second semester of 2014. In this research the relationship between the development of TEPCE and the application in the classroom is analyzed, how it facilitates planning and evaluation processes and its impact on the teaching/ learning process of children in early education. The method used was a descriptive qualitative approach which allowed the design of data collection instruments appropriate to gather information to analyze the phenomenon according to the reality studied, among them is the interview, observation and focus group. Upon completion of this study it was found no association between methodological strategies aimed at the development of TEPCE and their implementation in the classroom, because in the TEPCE new strategies are not being directed. Also, a conclusion was reached that the main drawback in the development of TEPCE is time, because in the time allotted for its development other activities occur.
\end{abstract}

Keywords: Teaching, Learning, TEPCE, methodological strategies, applicability. 


\section{INTRODUCCIÓN}

Los TEPCE son espacios de reflexión acción donde los docentes deben tomar decisiones de aspectos relevantes de la puesta en marcha del currículo nacional básico. En la práctica no se desarrollan tal y como están diseñados, ni cumplen el propósito para el cual fueron destinados.

Durante los encuentros no se ha manifestado el interés por mejorar la práctica educativa desde los TEPCE. Lo que fue de interés para realizar este trabajo de investigación, en el que se analizó la relación que existe entre el desarrollo de los TEPCE y la aplicación en el aula de clases, cómo facilita los procesos de planificación y evaluación y además como estos inciden en el proceso de enseñanza aprendizaje de los niños y niñas de Educación Inicial.

Dadas las problemáticas encontradas y la necesidad de mejorar las prácticas educativas, se consideró necesario investigar acerca de esta temática que es de relevancia y de muy poco conocimiento tanto de los docentes que participan como de la sociedad en general.

Los Talleres de Evaluación, Programación y capacitación Educativa (TEPCE), no son un componente separado del nuevo currículo de la educación general básica y media, son parte fundamental del mismo y su desarrollo es básico para la puesta en marcha y el cumplimiento del currículo.

\section{MATERIALES Y MÉTODOS}

La presente investigación corresponde al paradigma cualitativo, conocido también como investigación naturalista o fenomenológica, en este se describen paso a paso los procesos que fueron claves para la realización de este trabajo; por su alcance es de tipo descriptiva ya que se hace una descripción de los hechos y fenómenos que suceden tanto en el desarrollo de los TEPCE, como en el aula de preescolar, por el tiempo de realización es de corte transversal porque se desarrolló en un tiempo específico, en este caso en el segundo semestre del año 2014.

Se seleccionó el enfoque cualitativo descriptivo, como señala (Sampieri, Collado, \& Babtista, 2005) este enfoque presenta las características apropiadas para desarrollar este tipo de temas porque le permite al investigador involucrarse con las personas estudiadas y conocer desde sus ambientes la realidad en la que se desarrolla el fenómeno, esto le permite al investigador tener un abanico de oportunidades para estudiar el tema propuesto, ya que se puede establecer una relación cercana con las personas estudiadas y de este modo interpretar de una manera flexible la información de acuerdo al tema en estudio.

\section{Muestra y criterios de selección}

La selección de la muestra se hizo con base a criterios establecidos, con el propósito de garantizar la participación de aquellas personas que realmente aportarían con su experiencia y conocimientos, para entender el fenómeno, y así encontrarle respuesta a las cuestiones de investigación planteadas.

Para la investigación se seleccionó el preescolar Janeth Rodríguez porque es el preescolar más grande del municipio, está ubicado en el área urbana, es de fácil acceso y presenta todos los niveles (I. II y III nivel).

Para la selección de la maestra se tomó en cuenta la experiencia y el grupo, esta maestra tiene 30 años de dar preescolar y el grupo que tiene a su cargo es multinivel y por tanto reunía las características necesarias que aportarían información clave para el proceso de investigación.

Para seleccionar las maestras participantes en el grupo focal se tomó en cuenta maestras que fueran 
de Preescolares formales, comunitarios o privados, además que tuviera conocimiento de la modalidad preescolar y que asistan al TEPCE.

Para la selección de la asesora pedagógica se seleccionó directamente a la que atiende la modalidad de Educación Inicial.

Para la realización de este trabajo y recolección de la información se decidió utilizar los métodos de análisis, síntesis y deducción, así como el empleo de las siguientes técnicas empíricas de investigación:

Los instrumentos seleccionados fueron: la entrevista, observación, y el grupo focal, estas técnicas permitieron abordar con más detalle los aspectos que se propusieron analizar según las preguntas planteadas y los objetivos de investigación.

\section{Entrevista}

En esta investigación la entrevista fue aplicada a la asesora pedagógica que atiende Educación Inicial en el municipio de Pueblo Nuevo y a la maestra de multinivel del preescolar Janeth Rodríguez, la aplicación de entrevistas en esta investigación fue muy valiosa porque permitió recopilar información detallada del desarrollo de los TEPCE y como se aplica realmente en el aula de clases

\section{Observación}

Esta técnica fue aplicada mediante su respectivo instrumento en dos momentos en un primer momento se observó el desarrollo de los TEPCES con las maestras de preescolar del municipio y segundo se observó el aula de clases del III nivel del preescolar Janeth Rodríguez, esta técnica fue muy valiosa porque permitió recabar información muy valiosa en cuanto a las orientaciones que se dan en el desarrollo de los TEPCES y como se aplican realmente en el aula de clases.

\section{Grupo focal}

Esta técnica sirvió para comprender los conocimientos, actitudes y valores de cada uno de los docentes con respecto al tema de estudio. Con esta técnica se estableció un diálogo donde los docentes fueron dando su punto de vista de manera dinámica, participativa y motivadora donde sus aportes sirvieron de insumos al momento de hacer el análisis intensivo. Esta técnica es usada frecuentemente por los investigadores en grupos pequeños, seleccionados mediante un muestreo de tipo cualitativo elegido a criterio del investigador. El objetivo de la técnica consistió en profundizar sobre la aplicabilidad de los Talleres de Evaluación Programación y Capacitación Educativa en el desarrollo del proceso de Enseñanza y Aprendizaje.

La información obtenida en este trabajo investigativo se procesó mediante un sistema de categorías y subcategorías, tablas de reducción de la información por instrumentos los que se analizaron de manera cualitativa

Las etapas que se desarrollaron en el proceso de esta investigación permitieron recolectar información para darle continuo seguimiento a este proceso investigativo.

\section{Fase de Planificación o Preparatoria}

Para la realización de este trabajo investigativo primeramente se escogió el tema para trabajar, después de analizar se decidió trabajar con el tema: Desarrollo de los Talleres de Evaluación, Programación y Capacitación Educativa TEPCES y su aplicabilidad en el aula del multinivel del preescolar Janeth Rodríguez, en el municipio de Pueblo Nuevo.

Se seleccionó este tema porque es de gran relevancia para la Educación Infantil en este municipio ya que los TEPCES son un componente fundamental del currículo educativo nacional y de la buena aplicación de estos depende el éxito de la educación en las aulas de clases este país. 
En esta etapa se recopiló toda la información posible de diferentes medios, primeramente se visitó la biblioteca de la FAREM-ESTELI, para buscar toda la información posible que permitiera el conocimiento del tema a investigar luego se consultaron programas de estudio, afiches, periódicos y consultas en internet, para ampliar el proceso de investigación.

\section{Fase de Ejecución o Trabajo de Campo}

El trabajo de campo de la presente investigación consistió en aplicar instrumentos para esto primeramente se habló con el delegado municipal del Ministerio de Educación del municipio de Pueblo Nuevo para solicitar el permiso, luego con la asesora pedagógica que atiende Educación Inicial en el municipio para solicitar su colaboración en el proceso de la investigación, luego de obtener la aprobación se procedió a aplicar instrumentos a los informantes seleccionados.

\section{Informe Final o Fase Informativa}

La elaboración del documento se realizó paso a paso, conforme a los requerimientos del departamento de investigación de FAREM- ESTELI, primeramente el tema, luego los objetivos, marco conceptual y así cada parte que conforma el documento.

\section{Descripción de los TEPCE}

Los Talleres de evaluación, Programación y Capacitación educativa (TEPCE) se realizan todos los últimos viernes de cada mes, regidos por el Ministerio de Educación, tomando en consideración la Estrategia Nacional de Educación y fortaleciendo así las políticas educativas. Los docentes señalan que son de mucha importancia ya que nos sirven para auto prepararnos, intercambiar experiencias y llevar un mejor control de contenidos.

Es importante señalar que los docentes entrevistado consideran que los TEPCE inciden positivamente en el proceso de enseñanza aprendizaje, ya que se planean una serie de estrategias mucha importancia, lo que hace falta en el seguimiento y control para que sean aplicadas, logrando obtener aprendizajes significativos en con sus estudiantes.

\section{RESULTADOS Y DISCUSIÓN}

Después de procesar los instrumentos se conocieron los resultados los que se muestran según los propósitos de la investigación.

Identificar las estrategias didácticas que utiliza el docente en el aula de tercer nivel, según las orientaciones recibidas en los Talleres de evaluación programación y capacitación educativa.

Los TEPCE fueron creados con el propósito de desarrollar estrategias que luego fueran puestas en práctica en las aulas de clase para mejorar el proceso de enseñanza aprendizaje al realizar la observación, se pudo apreciar que se desarrollan pocas estrategias para desarrollar temas de Educación Inicial, debido a que las programaciones de contenidos ocupan la mayoría de tiempo, al momento de observar el desarrollo de las clases se percibió que la maestra desarrolla por su cuenta estrategias que permiten la facilitación del proceso de enseñanza aprendizaje, esto debido a su experiencia, pero que casi no se corresponden con estrategias planteadas en los TEPCE.

Todas las fuentes y/o informantes estuvieron de acuerdo que los TEPCE inciden en la aplicación de estrategias en las aulas de clases, porque en el desarrollo de estos, se comparten entre ellas logros, dificultades, nuevas propuestas metodológicas y como desarrollar temas difíciles, pero en la observación realizada al desarrollo de los TEPCE, no se identificó a ninguna maestra que compartiera con sus compañeras estrategias nuevas, tampoco se escuchó a ninguna de las maestras presentes que hablara sobre sus dificultades en el aula de clases, en el momento de la programación todas las maestras se dedican a transcribir del programa, por lo antes descrito se considera que en la práctica 
no se cumple lo que las informantes dicen, por tanto los TEPCE no están cumpliendo con su propósito original, ya que no se aprovechan estos espacios para los fines establecidos, esto conlleva a que a la hora de impartir clases las maestras no tengan nuevas metodologías para desarrollar sus clases, esto a su vez no permite que el aprendizaje sea de calidad.

Establecer la relación que existe entre la programación realizada en los Talleres de evaluación, Programación y Capacitación educativa, con la planificación diaria, y evaluación realizada por el docente en el preescolar

Según lo expuesto por las profesoras de Educación Inicial participantes en TEPCE y la docente del aula del preescolar Janeth Rodríguez, permite darse cuenta que el desarrollo de estos beneficia el proceso de planificación diaria, demostrando así la funcionabilidad que tienen estos Talleres, ya que de la programación que se realiza ese día se desprende la planificación diaria que realizan las docentes durante todo el mes, las maestras consultadas refirieron que con la programación realizada se les hace mucho más fácil su trabajo de planificación, además les permite la auto preparación, pueden llevar secuencia lógica de los temas y contenidos y control del tiempo que van a utilizar en el desarrollo de cada temática.

Una planificación ordenada de acuerdo a la programación abona a un aprendizaje de calidad en los niños y niñas de Educación Inicial, ya que esto le permite a la docente facilitar una clase dinámica, creativa que permite la integración de todos en las actividades desarrolladas en el aula de clases. Mediante la observación quedó demostrado que los planes diarios de la docente están plenamente articulados con los de la programación realizada para el mes correspondiente.

Con respecto a la evaluación, durante la observación realizada al desarrollo del TEPCE, no se encontró ninguna orientación en cuanto a evaluación de contenidos en niños y niñas de Educación Inicial, aun teniendo este un componente de evaluación.

\section{Determinar las ventajas $\mathbf{y}$ desventajas que tienen} los Talleres de Evaluación, Programación y Capacitación Educativa (TEPCE), para el proceso de enseñanza aprendizaje en el aula multinivel de

\section{Educación Inicial del preescolar}

Las maestras consultadas sobre este tema coinciden en que los TEPCE, tienen muchas ventajas en el proceso de enseñanza aprendizaje, porque son espacios muy importantes donde se les ofrece la oportunidad de compartir nuevas estrategias para mejorar la calidad del proceso enseñanza aprendizaje, también pueden exponer las dificultades que tienen sobre las formas de impartir un tema para así encontrar la solución por medio de la buena comunicación que se logra entre compañeras, además pueden evaluar como estuvo el mes anterior y tener unificación de criterios con respecto a un tema.

También desarrollan capacitaciones sobre temáticas importantes de Educación Inicial, esto es el propósito principal de los TEPCE como lo menciona Miguel de Castilla Urbina: (Urbina, 2014) Los TEPCE son Talleres mensuales de Programación, capacitación y de evaluación, en los que participan docentes, cuyo propósito es evaluar el cumplimiento de los objetivos de aprendizaje y de los programas de estudio del mes anterior, a fin de reflexionar sobre las causas que facilitaron o impidieron el aprendizaje de los estudiantes y tomar decisiones al respecto, y programar los objetivos, competencias y contenidos a desarrollar en el mes siguiente.

Los procesos descritos por De Castilla no se lograron percibir durante el desarrollo, esto se atribuye a la falta de tiempo, como lo mencionaba la asesora pedagógica. Además los maestros cumpliéndose el horario proceden a retirarse del centro hayan o no concluido. 
Las maestras consultadas coincidieron que la mayor dificultad que se presenta es que quitan mucho el tiempo que está asignado para el desarrollo de estos, con otras actividades como orientaciones generales y algunas charlas de otros temas como salud y seguros de vida, debido a esta situación no se pueden desarrollar muchas actividades que están contempladas en la agenda, cambiando así la organización, cuando esto sucede se reduce el tiempo que se dedica a la programación.

Las maestras también expresaron que tienen dificultades en la aplicación ya que con la nueva transformación curricular se les dificulta la planificación, debido a que se llevan mucho tiempo, porque los nuevos programas traen muchas actividades, además expresaron que no es fácil la aplicación de estrategias que reciben en los TEPCE en el aula de clases, debido a que en los preescolares no hay información actualizada.

Con la observación realizada al proceso de desarrollo de los TEPCE, se comprobó que algunas de las maestras presentaban problemas en la realización de sus programaciones debido a que muchas de ellas son educadoras comunitarias y no tienen el nivel adecuado, el nuevo currículo de preescolar es único tanto para preescolares comunitarios, como para preescolares formales. Los factores antes descritos no permiten que los TEPCE se desarrollen con su propósito original como expone Miguel De Castilla.

\section{CONCLUSIONES}

Basados en los análisis de resultados de esta investigación se puede concluir lo siguiente:

Existe poca correspondencia entre las orientaciones programadas en el TEPCE y las desarrolladas por la maestra en el aula, ya que la mayoría de las actividades desarrolladas por la maestra son de su propia iniciativa e innovación. Esto sucede porque las estrategias orientadas durante la programación no están relacionadas con los temas proyectados durante el mes, situación que lleva a la docente a retomar otras.

El desarrollo de los TEPCE beneficia el proceso de planificación diaria, ya que de la programación que se realiza ese día se desprende la planificación diaria que realizan las docentes durante todo el mes, la programación realizada les hace mucho más fácil el trabajo de planificación, además les permite la auto preparación, pueden llevar secuencia lógica de los temas y contenidos y control del tiempo que van a utilizar en el desarrollo de cada temática.

En el desarrollo del TEPCE, no se encontró ninguna orientación en cuanto a evaluación de contenidos en niños y niñas de Educación Inicial, en cambio la maestra si aplica las que se orientan en el documento curricular manual de planeamiento didáctico. Aunque la maestra retome las orientadas en el manual, no existe relación entre las estrategias desarrolladas en el TEPCE y las aplicadas por la docente en el aula.

Las maestras consultadas coincidieron que los TEPCE tienen grandes beneficios para el desarrollo de nuevas estrategias, pero en observación realizada no se demostró que se implementaran.

Las mayores dificultades en cuanto al desarrollo de los TEPCE son: el tiempo asignado, ya que este lo utilizan para otras actividades como orientaciones generales, charlas que no son de educación (seguros, salud), esto le resta tiempo a la programación y demás actividades propias del TEPCE, además algunas maestras tienen dificultad en la planificación y programación con el nuevo currículo, debido a su nivel académico, ya que con el nuevo currículo planean de la misma forma maestras comunitarias y maestras formales. 


\section{RECOMENDACIONES}

Después de conocer las conclusiones a las que se llegaron con la investigación se recomienda lo siguiente:

\section{Docente de Aula:}

Compartir con sus compañeras en el desarrollo del TEPCE las experiencias en cuanto a las estrategias que implementa en el aula, tanto para desarrollar las clases como para evaluar a las niñas y niños, para que las otras maestras conozcan cómo le ha funcionado y se pueda cumplir el propósito de los TEPCE.

Como docente con experiencia e ideas innovadoras, disponerse a colaborar con sus compañeras de TEPCE, en función de mejorar la calidad del proceso de enseñanza - aprendizaje.

\section{Asesora pedagógica:}

Aprovechar los espacios de los TEPCE, para desarrollar capacitaciones sobre la implementación de nuevas estrategias metodológicas retomando los temas de la programación que se realiza para ese mes, tanto para desarrollar las clases de los contenidos programados como para evaluarlos en el aula de clases.

Retomar las experiencias de maestros como la que se observó para facilitar procesos de capacitación.

\section{MINED:}

Abrir otros espacios para desarrollar otras actividades que non son propias del TEPCE y así garantizar el tiempo necesario para que este cumpla con sus propósitos.

\section{Otras maestras:}

Aprovechar los espacios como reuniones, capacitaciones, círculos pedagógicos y los mismos TEPCE para exponer sus dificultades en cuanto a planificación, evaluación y estrategias metodológicas.

\section{BIBLIOGRAFÍA}

Castilla, M. d. (10 de julio de 2014). El nuevo diario. com.ni. Obtenido de El nuevo diario.com.ni: http://www.elnuevodiario.com.ni/opinion/324411tepces-nuevo-modelo- curricular-nicaragense

De Castilla, M. (2009). NICARAGUA EDUCA. Recuperado el 21 de OCTUBRE de 2014, de NICARAGUA EDUCA: http://www. nicaraguaeduca.edu.ni/

Sampiery, R., Collado, C. F., \& Babtista, L. P. (2005). METODOLOGIA DEL LA INVESTIGACION. MEXICO DF: MWRAWHILL INTERAMERICANA.

Urbina, M. D. (2009). ¿Qué son los TEPCES? En M. D. Urbina, EL PLANEAMIENTO DIDÁCTICO $Y$ LA EVALUACIÓN DE LOS APRENDIZAJES (pág. 49). MANAGUA: SERIE EDUCATIVA:.

Urbina, M. D. (10 de julio de 2014). El nuevo diario. com.ni. Obtenido de El nuevo diario.com.ni: http://www.elnuevodiario.com.ni/opinion/324411tepces-nuevo-modelo- curricular-nicaragense Urbina, M. D. (09 de octubre de 2014). Miguel De Castilla Urbina. Obtenido de Miguel De Castilla Urbina: http://migueldecastilla.blogspot.com/ 